\title{
Charge fluctuations in single-electron tunneling oscillations
}

\author{
C. Negri $^{1}$ and F. Pistolesi ${ }^{1}$ \\ ${ }^{1}$ Univ. Bordeaux, LOMA, UMR 5798, F-33400 Talence, France. \\ CNRS, LOMA, UMR 5798, F-33400 Talence, France.
}

(Dated: June 15, 2018)

\begin{abstract}
It has been predicted that in the presence of a sufficiently high-dissipative environment transport in a small tunnel junction can become extremely regular, giving rise to the phenomenon of singleelectron tunneling oscillations. Recent progress in detection of high-frequency current fluctuations and the interest in single-electron sources motivate further investigations on the expected accuracy of the charge oscillations as a function of the impedance of the environment. In this paper we study theoretically the charge-fluctuation spectrum at finite frequency for the system at hand, and investigate its behavior as a function of the external impedance. The evolution and the disappearance of the single-electron oscillations peak is described by analytical and numerical methods.
\end{abstract}

\section{INTRODUCTION}

Coulomb blockade of voltage-biased tunnel junctions in the presence of a dissipative environment is a wellunderstood phenomenon of quantum transport (for a review see for instance Ref. [1]). It has been shown that if the impedance of the environment $R$ is smaller than the tunnel junction resistance $R_{t}$, but larger than the quantum of resistance $R_{Q}=2 \pi \hbar / \mathrm{e} \approx 25.8 k \Omega$ ( $\hbar$ being the reduced Planck constant and e the electron charge), the current is suppressed for bias voltage $V$ smaller than the Coulomb gap e/2C, associated with the capacitance $C$ of the junction ( $C$ is typically in the $\mathrm{fF}$ range). This effect can be visible for temperatures lower than the Coulomb charging energy $\mathrm{e}^{2} / 2 C$ and for $R_{t} \gg R_{Q}$, which assures suppression of cotunneling. In practice realizing a highimpedance environment with a flat frequency response till frequencies of the order of $\mathrm{e}^{2} / 2 C \hbar$ is a challenging experimental problem, as discussed in detail in Ref. [2]. Most experimental observations of Coulomb blockade phenomena in single tunnel junctions are actually done in an intermediate-impedance situation $\left(R \sim R_{Q}\right)$, which leads at least to clearly non-linear characteristics of the junction; see for example Refs. [3, 4]. Nevertheless, it is feasible to realize impedances of the order of some hundreds of $\mathrm{k} \Omega$; for instance in the recent Ref. [5] a resistance $R \sim 0.4 \mathrm{M} \Omega$ has been realized. For these values not only the suppression of the current at low-bias voltage should be visible, but also an appealing effect predicted in the 1980s [6] 9]: the single-electron tunneling oscillations (SETOs).

The idea behind this effect can be understood in the simplest way in the limit $R, V \rightarrow \infty$ with $V / R=I_{b}$, so that the tunnel junction is current biased. The current slowly charges the capacitance. When $V=Q / C$ reaches the threshold e/2C one electron can cross the junction. If $I_{b} \ll \mathrm{e} / R_{t} C$ this will happen just after $V$ has reached the threshold. The charge on the capacitance after the tunneling event will be $Q \simeq-\mathrm{e} / 2$, and $Q$ will start to slowly increase again. A time $\sim \mathrm{e} / I_{b}$ is needed before a new electron can cross the junction and the sequence can start again. The voltage at the junction will thus be periodically modulated at a tunable frequency $\sim I_{b} /$ e.

Observation of this phenomenon is difficult. The implementation of the required strong-impedance environment has been realized by different authors using onchip resistors 10 16. An alternative approach has also been tried by designing the environment with tunneljunction arrays [17, 18], exploiting the fact that a large number of arrays reduces the stochastic nature of the current 19]. Recently, observation of soliton-like singleelectron oscillations with this method has been reported [20]. A similar phenomenon for superconducting Josephson junctions has been predicted (Bloch oscillations) 21] and investigated by many authors [22 25]. Arrays of dc SQUIDs have also been exploited in this case to build up the proper environment to obtain Coulomb blockade of Cooper pairs 26 28]. Reports on the observation of Shapiro-step-like structures in microwave-irradiated junctions constitute the present state of the art for the experimental probe of this effect [14, 23, 29].

Progress in the detection of high-frequency current fluctuations 30 33. can open new possibilities of observation of this phenomenon and of the crossover region, where the oscillations are not completely established. At the same time the possibility of generating a periodic and frequency-tunable electric signal without any oscillating source is an interesting opportunity and could have applications, for instance, as a motion actuator in nanomechanical systems or a controlled single-electron source.

It should be mentioned that Coulomb blockade is instead easily observed in double tunnel junctions even in absence of an environment. This happens since the second tunnel junction plays the role of the large-impedance environment suppressing quantum charge fluctuations and preventing tunneling if the voltage is below a threshold. Note however that this configuration (without environment) will not give rise to single-electron oscillations, but to the well known sequential transport regime. Electrons hop stochastically through the first and then the second junctions: The advantage of an ohmic environment is that it can generate (at least in principle) a stable current source.

In this paper we study how accurate the SETOs can be 


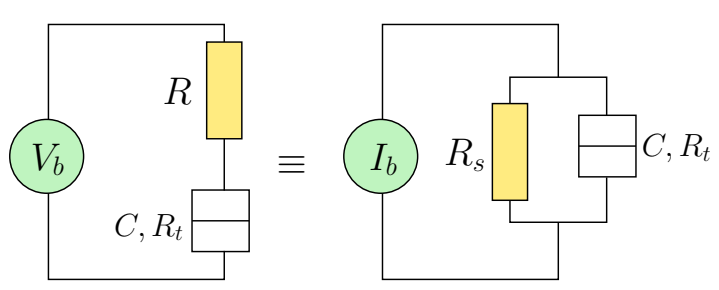

FIG. 1. Circuit scheme of the system considered: a tunnel junction with capacitance $C$ and tunneling resistance $R_{t}$, biased by the constant current $I_{b}$ and shunted by a resistance $R_{s}$ in parallel (right), which is equivalent to a junction biased by a voltage $V_{b}$ and with a resistance $R$ in series (left) provided that $V_{b}=I_{b} R$ and $R=R_{s}$.

as a function of the impedance and of the bias conditions. In order to do this we will study the charge-fluctuation spectrum at the junction capacitance (or equivalently the current-fluctuation spectrum through the resistance load), and in particular the width of the peak at the frequency $\sim I_{b} /$ e. Notwithstanding the relatively large number of papers on this subject, some specifically addressing the dynamics of arbitrarily biased mesoscopic tunnel junctions with an analytical approach [34 36], a consistent calculation of these quantities is not available. It can be useful in order to evaluate the expected effect in view of measuring current fluctuations in this kind of device. In this paper we show that the width of the peak scales as the inverse of the impedance for large $R / R_{t}$. The peak remains observable till values of $R / R_{t}$ of the order of 5 .

The plan of the paper is the following. In Sec. II we present the model describing the transport through the junction. In Sec. III we discuss the different regimes that the system undergoes by varying the bias voltage and the environment resistance. In particular we obtain an analytical expression for the the $I-V$ characteristic in the SETOs regime. In Sec. IV we calculate analytically the charge spectrum. The results are discussed and compared with numerical Monte Carlo simulations in Sec. V Section VI gives our conclusions.

\section{THE SYSTEM AND THE MODEL}

Let us consider a tunnel junction with tunneling resistance $R_{t} \gg R_{Q}$ and associated capacitance $C$. The circuit is voltage biased (at voltage $V_{b}$ ) at zero temperature $(T=0)$ in the presence of a resistor of resistance $R$ in series with the junction (see Fig. (10), left side). This circuit is equivalent to one with a current source $I_{b}$ and a shunt resistor $R_{s}$ in parallel to the junction, provided $I_{b}=V_{b} / R$ and $R=R_{s}$ (see Fig. (11), right side). We will thus use the parallel configuration to describe the device in analogy with the previous literature [7]. It is clear that the results can be readily converted to the voltage bias case. In particular note that the limit $R_{s} \rightarrow \infty$ (specifically $R_{s} \gg R_{t}$ ) describes the ideal current source.
Since we are interested in studying SETOs we need $R_{s} \gtrsim R_{t} \gg R_{Q}$. We thus assume from the outset that $R_{s} \gg R_{Q}$, which allows to neglect quantum fluctuations and treat the charge degrees of freedom classically [7]. We also assume that the environment has a flat frequency response $Z(\omega)=R_{s}$ up to frequencies $\hbar \omega \approx \mathrm{e}^{2} / 2 C$. This hypothesis, though not easy to fulfill in practice, is the common assumption in the literature about this problem, and allows a simpler and more transparent approach. In this regime transport through the junction is described by the theory currently known as orthodox Coulomb blockade theory. Specifically the electron-tunneling rate depends on the voltage at the junction $\left(V_{J}\right)$ as follows:

$$
\Gamma\left(V_{J}\right)=\theta\left(V_{J}-\mathrm{e} / 2 C\right)\left(V_{J}-\mathrm{e} / 2 C\right) /\left(\mathrm{e} R_{t}\right),
$$

where $\theta$ is the Heaviside function. [At finite temperature the function $\theta(V)$ is substituted by $1 /\left(e^{-\mathrm{e} V / k_{B} T}-1\right)$.] If $\left(R_{Q} \ll\right) R_{s} \ll R_{t}$ the standard picture of Coulomb blockade applies to the degrees of freedom of the environment: They have the time to relax to thermal equilibrium between two electron-tunneling events. The current-voltage characteristic in this case is then given by $I_{J}\left(V_{J}\right)=\left(V_{J}-\mathrm{e} / 2 C\right) \theta\left(V_{J}-\mathrm{e} / 2 C\right)$, exposing a clear Coulomb gap for the current $I_{J}$ through the junction. But if $R_{s} \sim R_{t}$ or larger (for moderate values of the bias voltage) the resistive environment (described for instance by a large collection of bosonic modes) reaches thermal equilibrium, but not the charge on the capacitance $Q(t)$, which needs a time $\tau_{s}=R_{s} C$ to relax to its stationary state. Formula (11) still holds, but with a time-dependent voltage $V(t)=Q(t) / C$. The time dependence of the charge is given by the solution of the differential equation:

$$
\dot{Q}=-Q / R_{s} C+I_{b}
$$

which for an initial condition $Q_{0}$ at $t=0$ reads

$$
Q_{f}\left(Q_{0}, t\right)=\left(Q_{0}-I_{b} \tau_{s}\right) e^{-t / \tau_{s}}+I_{b} \tau_{s} .
$$

The stochastic problem is then completely formulated and in the remainder of the paper we discuss the behavior of the current and of the charge as a function of the two relevant dimensionless parameters of the problem: $\rho=R_{s} / R_{t}$ and $\kappa=\left(I_{b}-I_{\mathrm{th}}\right) / I_{\mathrm{th}}$, with $I_{\mathrm{th}}=\mathrm{e} / 2 \tau_{s}$ the threshold for the current to start flowing through the tunnel junction.

The current through the junction has already been calculated numerically in the very early literature [8]. We obtained the same results by Monte Carlo simulation and, for convenience, we reproduce the curves in Fig. (2). The limit of infinite $R_{s}$, or ideal current source, was discussed in details in Refs. [7, 37]. There it was shown in particular that in this limit the system is in the SETOs regime with frequency $I_{b} / \mathrm{e}$ with an averaged voltage at the junction given by (see [7]):

$$
\left\langle V_{\mathrm{J}}\right\rangle=\sqrt{\frac{\pi \mathrm{e} R_{t}\left\langle I_{\mathrm{J}}\right\rangle}{2 C}} .
$$




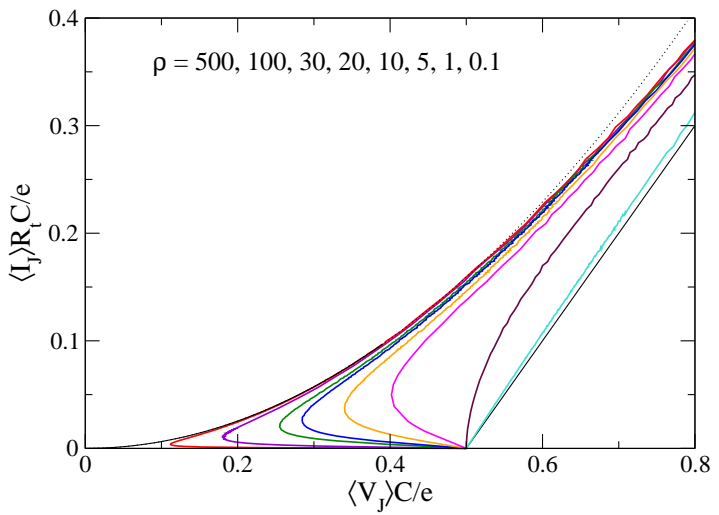

FIG. 2. (Color online) Average current through the junction versus average voltage for different values of the ratio $\rho=$ $R_{s} / R_{t}$. The curves evolve from the standard Coulomb blockade suppression (rightmost line corresponding to $\rho=0.1$ ) to a square root behavior (leftmost line $\rho=500$ ). The dotted line is the large- $\rho$ limit of Eq. (4).

In the next section we will discuss the behavior of the system for the intermediate regimes appearing when $R_{s} / R_{t}$ is not infinite, deriving in particular new analytical expressions for the SETOs frequency and $I-V$ characteristics.

\section{REGIMES OF CURRENT TRANSPORT}

In this section we study the evolution of the current through the junction as a function of the current bias for different values of the external resistance. The most interesting case is when $R_{s} / R_{t}$ is very large, we thus plot in Fig. 3 the current on a logarithmic scale for the extreme value of $R_{s} / R_{t}=5 \times 10^{2}$. Fig. (3) will be used as a "map" for the rest of the section, where we will discuss how the junction evolves through the four different regimes of transport indicated in the figure with roman numerals from (I) to (IV). Fig. (4) shows the behavior of $Q(t)$ in the different regimes.

\section{A. Non SETOs regimes}

Let us begin with the region indicated with (I) in Fig. (3). For $I_{b}<I_{\text {th }}$ the whole current flows through the shunting resistance and the voltage at the junction

$$
\left\langle V_{\mathrm{J}}\right\rangle_{I}=R_{s} I_{b}
$$

remains below the threshold of the Coulomb blockade: e/2C. Note that this first branch of the $I-V$ characteristic in Fig. (2) is flattened on the $I_{J}=0$ value and it is thus not visible.

For $I_{b}>I_{\text {th }}$ transport through the junction becomes possible. For $I_{b}-I_{\mathrm{th}} \rightarrow 0^{+}$one can identify a Poissonian regime of transport (region (II) in Fig. (3)), where the

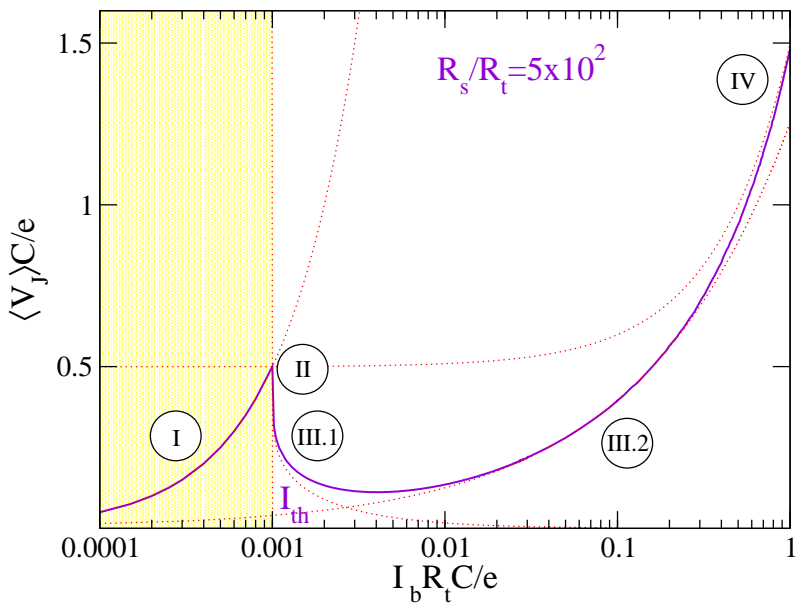

FIG. 3. (Color online) Average voltage through the junction versus bias current in logarithmic scale $\left(\rho=5 \times 10^{2}\right)$ : Four different transport regimes can be outlined. The solid line gives the results of the numerical simulations, while dashed lines represent the analytical curves given in Sec. III
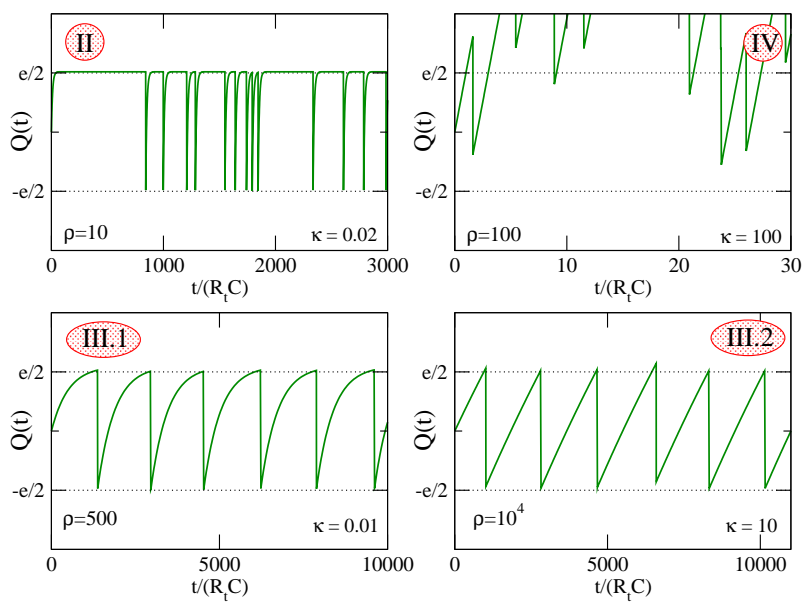

FIG. 4. (Color online) The time behavior of the charge in the different regimes. Extreme values of the parameters $\rho$ and $\kappa$ have been chosen to best outline the differences.

time between tunneling events fluctuates strongly. This is due to the fact that $\Gamma(V)$ as given by Eq. (1) vanishes linearly near the threshold, and for very small $I_{b}-I_{\text {th }}$ the charge has always the time (typically $\tau_{s}$ ) to reach the saturation value $Q_{\mathrm{s}}=I_{b} \tau_{s}$. The typical inverse time between two tunnel events is:

$$
1 / \tau_{\mathrm{eff}}=\Gamma\left(Q_{\mathrm{s}}\right)=\left(R_{s} / R_{t}\right)\left(I_{b}-I_{\mathrm{th}}\right) / \mathrm{e} \ll 1 / \tau_{s} .
$$

The last inequality sets also the region of existence of the regime (II) i.e. , $0<\kappa \ll 1 / \rho$. The average $V_{J}$ can be readily evaluated by averaging the oscillations of the charge $Q(t)=Q_{\mathrm{s}}-\mathrm{e} e^{-t / \tau_{s}}$ on the average time between two tunneling events $\tau_{\text {eff }}+\tau_{s} \approx \tau_{\text {eff }}$. This gives:

$$
\left\langle V_{\mathrm{J}}\right\rangle_{I I}=R_{s}\left[I_{b}\left(1-R_{s} / R_{t}\right)+I_{\mathrm{th}} R_{s} / R_{t}\right]
$$

and the curve is shown dashed in Fig. (3). Note that 
the slope changes sign at $R_{s} / R_{t}=1$. We will see that for $R_{s} \ll R_{t}$ this region joins continuously region (IV) without the appearance of region (III).

It is thus convenient to discuss now the region (IV) defined as the limit of large $I_{b}$. In this limit the junction has a $I-V$ characteristic of a normal resistor shifted by the Coulomb gap. The average $V_{J}$ reads then:

$$
\left\langle V_{\mathrm{J}}\right\rangle_{I V}=\frac{R_{s} R_{t}}{R_{s}+R_{t}}\left(I_{b}+\frac{\mathrm{e}}{2 R_{t} C}\right) .
$$

This expression holds for $\left\langle V_{\mathrm{J}}\right\rangle \gg \mathrm{e} / 2 C$, i.e. for $I_{b} / I_{\mathrm{th}} \gg$ $\left(R_{t}+R_{s}\right) / R_{s}$. For $R_{s} \gg R_{t}$ it is then clear that a large region defined by the condition

$$
1 / \rho \ll \kappa \ll \rho
$$

exists between region (II) and region (IV). This is the SETOs region, (III) in Fig. (3), which will be discussed below. On the other side, for $R_{s} \ll R_{t}$, one sees that region (II) and region (IV) overlap at $\kappa \approx 1$. Actually it is straightforward to check that Eq. (8) expanded to first order in $R_{s} / R_{t}$ coincides with Eq. (7).

\section{B. SETOs regime}

Let us now discuss the single-electron oscillations regime, defined as region (III) in Fig. (3). This region is present only if $R_{s} \gg R_{t}$ and is characterized by nearly periodic electron tunneling events, since the time between two events is dominated by the deterministic charging time of the capacitance. This time is typically of the order of $t_{\star}$, defined as the time needed to charge the capacitance from $Q=-\mathrm{e} / 2$ to $Q=\mathrm{e} / 2$ :

$$
t_{\star} / \tau_{s}=\ln \left(\frac{I_{b}+I_{t h}}{I_{b}-I_{t h}}\right)=\ln \left(\frac{2+\kappa}{\kappa}\right) .
$$

The electrons hop just after the threshold voltage has been reached.

A general statistical theoretical framework is presented in Ref. 7] (and recalled in the Appendix), but its analytical solution is given there only in the ideal current source limit $\left(R_{s} / R_{t} \rightarrow \infty\right)$. Actually in the case of the SETOs the approach is simplified and further progress is possible. In order to obtain these results and for the calculation of the correlation function of the next section it is convenient to introduce a few concepts. In Fig. (5) the typical time dependence of the charge $Q(t)$ in the SETOs regime is shown. We can associate a number $n$ to each hopping event and define $t_{n}$ and $\tau_{n}=t_{n}+\delta \tau_{n}$ as the instant of time when $Q(t)=\mathrm{e} / 2$ and when the hopping event takes places, respectively (see Fig. (5)). These quantities fluctuate randomly, but a correlation between $t_{n}$ and $t_{n-1}$ exists. Inversion of Eq. (3) gives the time needed to reach the border of the Coulomb blockade region starting from a charge $Q_{0}$ :

$$
\Xi\left(Q_{0}\right)=-\tau_{s} \ln \left(\frac{I_{b} \tau_{s}-\mathrm{e} / 2}{I_{b} \tau_{s}-Q_{0}}\right) .
$$

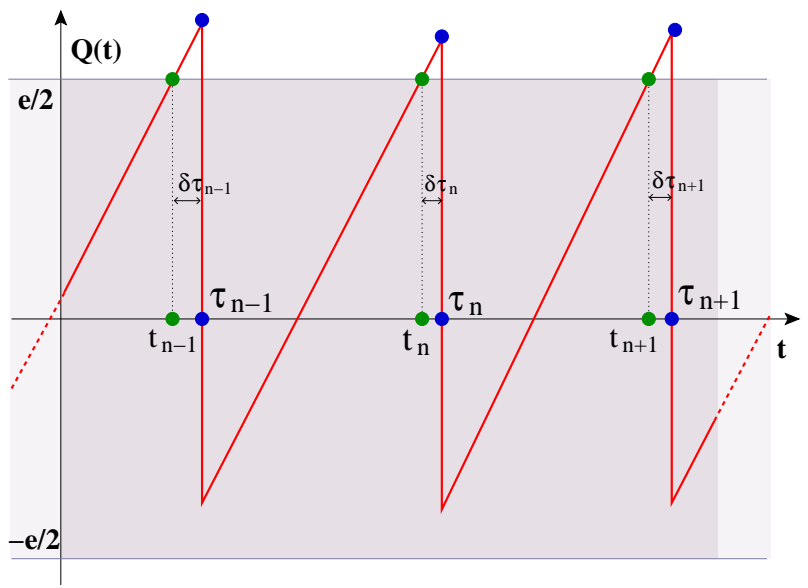

FIG. 5. (Color online) Details of the tunneling process: $t_{n}$ is the time at which the charge reaches the blockade region border after the $(n-1)$ th tunneling event and $\delta \tau_{n}$ is the time it stays outside the border before the $n$th event.

The following relation between successive times $t_{n}$ holds:

$$
t_{n}-t_{n-1} \equiv \mathfrak{F}\left(\delta \tau_{n-1}\right),
$$

with

$$
\begin{aligned}
\mathfrak{F}(\delta \tau) & =\delta \tau+\Xi\left(Q_{f}\left(\frac{\mathrm{e}}{2}, \delta \tau\right)-\mathrm{e}\right) \\
& =\delta \tau-\tau_{s} \ln \left(\frac{\kappa}{2+\kappa e^{-\delta \tau / \tau_{s}}}\right) .
\end{aligned}
$$

Let us now introduce the probability $P_{n}(t)$ that $n$ electrons have tunneled through the junction at the time $t$. Within the SETOs region, this quantity is different from zero only in a small time region of the order of $t_{\star}$, and, in particular, the above mentioned condition on the typical hopping time implies that $P_{n}(t)$ will reach 1 and then vanish in a time much shorter than $t_{\star}$, just after $Q(t)$ crosses the threshold e/2. The rate equation for $t \geq t_{n}$ $\left[Q\left(t_{n}\right)=\mathrm{e} / 2\right]$ takes the simple form:

$$
\frac{d P_{n}}{d t}(t)=-\Gamma\left(Q_{f}(\mathrm{e} / 2, t) / C\right) P_{n}(t)
$$

with the initial condition $P_{n}\left(t_{n}\right)=1$. The solution reads:

$$
P_{n}(t)=e^{-\frac{\left(I_{b} \tau_{s}-\mathrm{e} / 2\right)}{\mathrm{e} R_{t} C} \tau_{s}\left(\frac{t-t_{n}}{\tau_{s}}+e^{-\frac{t-t_{n}}{\tau_{s}}}-1\right)} .
$$

For short times $\left(t-t_{n} \ll \tau_{s}\right)$ it has a Gaussian form

$$
P_{n}(t) \approx \exp \left\{-\left(t-t_{n}\right)^{2} \kappa \rho /\left(4 \tau_{s}^{2}\right)\right\}
$$

with a decay time scale $\sim \tau_{s} / \sqrt{\kappa \rho} \ll t_{\star}$ in region (III). The Gaussian form will thus be used in the following for the analytical calculations.

From the knowledge of $P_{n}(t)$ it is possible to obtain the probability density that a hopping event takes places at time $t: \mathcal{P}(t)=-d P_{n} / d t$, for $t \geq t_{n}$. This allows us to calculate the average delay time $\langle\delta \tau\rangle$ for an electron 
to hop after the threshold e/2 has been crossed by the charge $Q(t)$ :

$$
\langle\delta \tau\rangle=\int_{0}^{\infty} d t t \mathcal{P}(t)
$$

In particular when $P_{n}$ can be approximated by the Gaussian (16) one obtains $\langle\delta \tau\rangle / \tau_{s}=\sqrt{\pi /(\kappa \rho)}$ with $\langle\delta \tau\rangle \ll t_{\star}$. To obtain the period of the SETOs one has to average the nonlinear expression (13): $\mathcal{T}=\langle\mathfrak{F}\rangle$, which again in the SETOs region simplifies to

$$
\mathcal{T}=t_{\star}+2\langle\delta \tau\rangle /(2+\kappa) .
$$

Let us now come back to the probability. Conservation of the probability gives that $P_{n+1}(t)=1-P_{n}(t)$. Since in this approximation the behavior is quasiperiodic, the charge on the capacitor for the $n+1$ electron is on average $Q_{f}\left(\mathrm{e} / 2, t-t_{n}-\mathcal{T}\right)$. (A more precise discussion on the validity of this last average can be found in the Appendix, where the problem is analyzed more rigorously.) The average charge can then be computed by averaging over a period as follows:

$$
\begin{aligned}
\langle Q\rangle=\int_{t_{n}}^{t_{n}+\mathcal{T}} \frac{d t}{\mathcal{T}} & {\left[Q_{f}\left(\frac{\mathrm{e}}{2}, t-t_{n}\right) P_{n}(t)+\right.} \\
+ & \left.Q_{f}\left(\frac{\mathrm{e}}{2}, t-t_{n}-\mathcal{T}\right) P_{n+1}(t)\right] .
\end{aligned}
$$

In the limit of $\rho \gg 1$ only the gaussian part of the probability is relevant, and the integral gives:

$$
\begin{aligned}
\langle Q\rangle= & Q_{\mathrm{s}}-\left(Q_{\mathrm{s}}-\frac{\mathrm{e}}{2}\right)\left(e^{\frac{\mathcal{T}}{\tau_{s}}}-1\right) \frac{\tau_{s}}{\mathcal{T}} \times \\
& \times\left[1-\sqrt{\frac{\pi}{\kappa \rho}} e^{\frac{1}{\kappa \rho}} \operatorname{Erfc}(1 / \sqrt{\kappa \rho})\right] .
\end{aligned}
$$

Eq. (20) leads to $\left\langle V_{J}\right\rangle_{I I I}=C\langle Q\rangle$. With little loss in the accuracy Eq. (20) can be simplified to the form:

$$
\langle Q\rangle \approx \frac{\mathrm{e}}{2}\left[\kappa+1-2\left(1-\sqrt{\frac{\pi}{\kappa \rho}}\right) / \ln \left(\frac{2+\kappa}{\kappa}\right)\right] .
$$

The analytical expressions (20), (21), and (A8) obtained in the appendix, are compared to the Monte Carlo results in Fig. (6). These expressions describe the current with good accuracy, and in particular they all capture the presence of a minimum in the voltage $V_{J}$. This minimum signals the crossover region between two different kinds of SETOs. We indicate them in Fig. (3) as III.1 and III.2. The latter appears for $1 \ll \kappa \ll \rho$. In this case, and in the extreme limit $\rho \rightarrow \infty$ the SETOs period becomes $\mathcal{T}=\mathrm{e} / I_{b}$, i.e. corresponds exactly to the time needed to the ideal current source to furnish a charge e. The saturation value for the charge $\left(Q_{s}\right)$ in this regime is much larger than e/2, implying that only the linear part of the exponential in Eq. (3) is explored. This is important since the small fluctuations in the hopping times do not affect the evolution equation for the next electron.

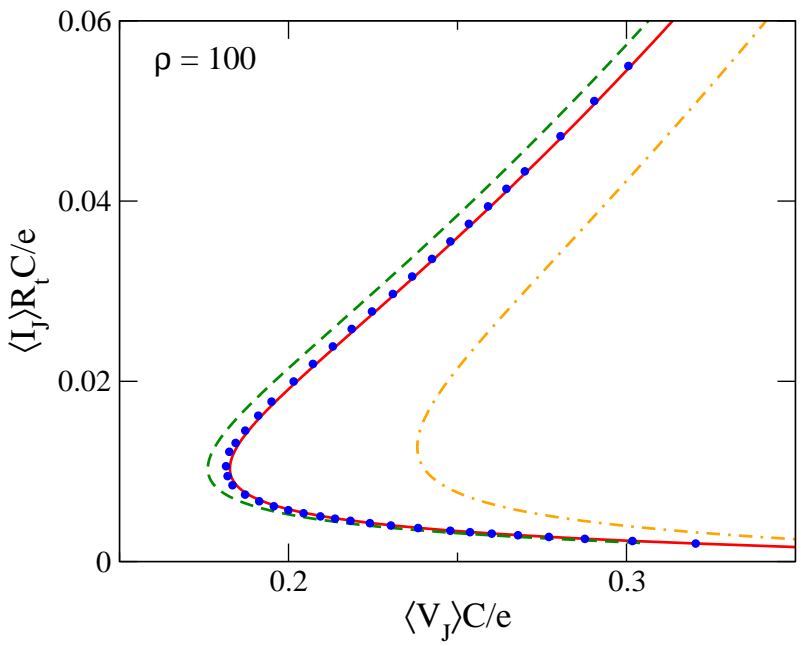

FIG. 6. (Color online) Comparison between the different approximations for the SETOs "nose" of the currentvoltage characteristics: Monte Carlo data (circles) are shown against analytical calculations from Eq. (A8) (solid), Eq. (20) (dashed), and Eq. 21) (dot-dashed).

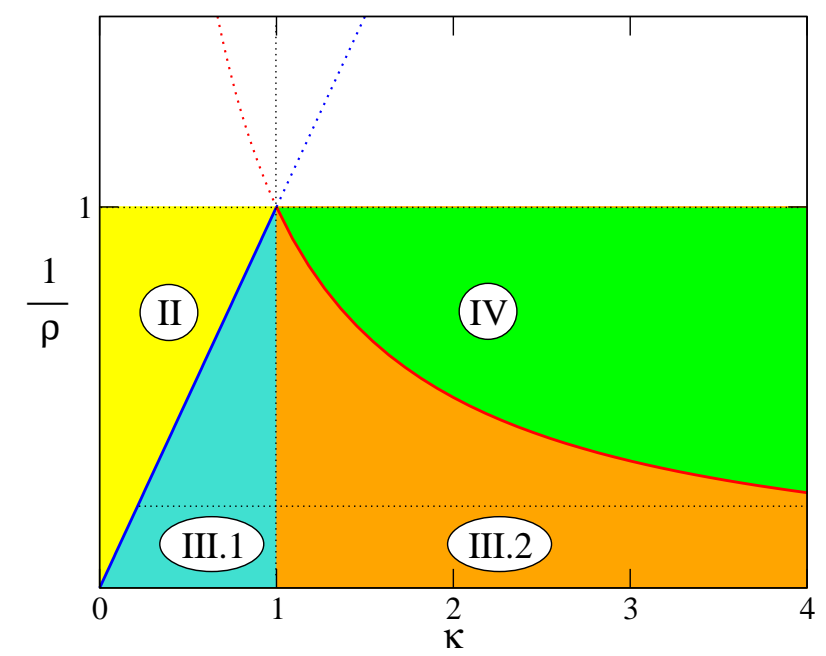

FIG. 7. (Color online) Scheme of the boundaries of the transport regimes as a function of the relevant parameters: The tunneling/shunt resistances ratio $1 / \rho=R_{t} / R_{s}$ and the relative distance of the current bias from the threshold $\kappa=$ $\left(I_{b}-I_{\mathrm{th}}\right) / I_{\mathrm{th}}$. The SETOs regime exists only in the limit $\rho \gg 1$.

One can readily verify that in the limit of an ideal current source the charge time dependence around each $t_{n}$ is $Q(t)=I_{b}\left(t-n \mathrm{e} / I_{b}\right)$. The non-linear corrections instead add a stochastic dependence on the time evolution of the charge. The period, for instance, does not depend on $\langle\delta \tau\rangle$ anymore for $\kappa \rightarrow \infty$, as is clear from Eq. (18). This extreme limit is not realistic and thus the second term given in (18) is normally important.

Reducing the current bias, the saturation charge $Q_{s}$ becomes of the order of e/2 $\left(\kappa=2 Q_{s} / \mathrm{e}-1<1\right)$ and the non-linear behavior of $Q(t)$ begins to correlate different 
hopping events. The charge time evolution in this regime is characteristic and resembles a shark fin, as shown in Fig. (4). It is also clear from (18), that in this regime the stochastic fluctuations have the greatest impact on the average SETOs period.

These two regimes can be identified on the current plot Fig. (6) and in the analytical expression (21) as the two branches joined by a minimum of the voltage. The large bias behavior $(\kappa \gg 1)$ of (21) gives e $\sqrt{\pi \kappa / \rho} /(2 C)$, which is the Averin-Likharev expression for the current (4) $\left(\left\langle I_{J}\right\rangle \sim I_{b}\right.$ in this limit), while in the opposite limit the long exponential charging time is dominating: $\left\langle V_{J}\right\rangle \approx(\mathrm{e} / C) \sqrt{\pi} /(\sqrt{\kappa \rho} \ln (2 / \kappa))$.

The overall situation is summarized in Fig. (7). Since it is very difficult in practice to experimentally reach large values of $\rho$, the plot in Fig. (7) suggests that a good experimental choice can be $\kappa=1$, for which the SETOs appear for the lowest values of $\rho$. We will discuss in the following the correlation function of the charge in order to analyze quantitatively the evolution of the accuracy of the SETOs.

\section{CHARGE-FLUCTUATION SPECTRUM}

A quantitative measure of the accuracy of the SETOs is given by the time correlation of the charge. This can be defined as

$$
S(\tau)=\langle Q(t+\tau) Q(t)\rangle-\langle Q(t+\tau)\rangle\langle Q(t)\rangle,
$$

where the average is performed over a statistical ensemble and the result does not depend on $t$, since the stochastic process is stationary. Note also that this quantity is proportional to the spectrum of current fluctuations through the shunt resistance $R_{s}: S_{R}(\tau)=S(\tau) / \tau_{s}^{2}$. In the case of voltage-biased junction (see Fig. (10), $S_{R}$ gives the current fluctuations that can be directly measured through the load resistance $R$.

For perfectly periodic charge oscillations the Fourier transform of (22) is given by a sum of Dirac delta functions at $\omega=2 \pi n / \mathcal{T}$, with $n$ integer. The non-periodic fluctuations introduce a finite width of these peaks. The form of $S(\omega)$ measures thus directly and with a simple procedure the accuracy of the periodic charge transfer. In this section we derive an analytical expression for $S(\omega)$ that allows us to better understand the origin of the fluctuations. In the next section we will compare these results to those obtained numerically by Monte Carlo simulations.

In order to calculate the Fourier transform of $S(\tau)$ for this stationary process it is convenient to define the charge $Q(t)$ over a time $0<t<\Lambda$, with $\Lambda \gg \mathcal{T}$ so that many SETOs are present in a single sample of $Q(t)$. One can then calculate the Fourier series

$$
Q_{p}=\int_{0}^{\Lambda} \frac{d t}{\Lambda} Q(t) e^{i p \frac{2 \pi}{\Lambda} t} \quad, \quad Q(t)=\sum_{p} e^{-i p \frac{2 \pi}{\Lambda} t} Q_{p} .
$$

Substituting Eq. (23) into Eq. (22) and averaging over $t$ one obtains:

$$
S(\tau)=\sum_{p}\left\langle\left|Q_{p}\right|^{2}\right\rangle e^{-i p \frac{2 \pi}{\Lambda} \tau}-Q_{0}^{2},
$$

which can be used numerically to compute the correlation function from the Monte Carlo data, or analytically, by performing the limit $\Lambda \rightarrow \infty$. In particular the Fourier transform can be defined as

$$
S(\omega)=\int_{-\Lambda / 2}^{+\Lambda / 2} d \tau e^{i \omega \tau-0^{+}|\tau|} S(\tau),
$$

which gives

$$
S(\omega)=\sum_{p}\left(\left\langle\left|Q_{p}\right|^{2}\right\rangle-\delta_{p, 0} Q_{0}^{2}\right) 2 \pi \delta\left(\omega-\omega_{p}\right),
$$

with $\omega_{p}=2 \pi p / \Lambda$. The presence of the Dirac delta functions is an artifact due to the periodic extension induced by the Fourier transform. In practice, since the frequency scale $1 / \Lambda$ is infinitesimal one can obtain the smooth function $S(\omega)$ by averaging the expression (26) for each value of $\omega$ over a small interval $2 \pi / \Lambda$. This simply gives that

$$
S\left(\omega_{p}\right)=\Lambda\left\langle\left|Q_{p}\right|^{2}\right\rangle
$$

for $p \neq 0$ (Wiener-Khinchin theorem).

The problem is now reduced to the calculation of the Fourier series of the charge. Using the definitions of $t_{n}$ and $\tau_{n}$ given before in Eq. (11) and assuming that the extrema of the time evolution of $Q(t)$ coincide with the two hopping events at times $\tau_{0}$ and $\tau_{N}$ we can write:

$$
\begin{aligned}
Q_{p} & =\sum_{n=0}^{N-1} \int_{\tau_{n}}^{\tau_{n+1}} \frac{d t}{\Lambda} Q(t) e^{i \omega_{p} t}= \\
& =\sum_{n=0}^{N-1} e^{i \omega_{p} \tau_{n}} \int_{0}^{\tau_{n+1}-\tau_{n}} \frac{d t}{\Lambda} Q_{f}\left(\mathrm{e} / 2, t-t_{n+1}+\tau_{n}\right) e^{i \omega_{p} t}
\end{aligned}
$$

In the limit of well-established SETOs the integral gives a contribution that fluctuates very little. On the contrary the exponentials are much more sensitive to even small fluctuations of the tunneling times, since the phase results from the accumulation of many different hopping events. For this reason we expect that the upper integration limit can be substituted with the period of the SETOs $\tau_{n+1}-\tau_{n} \approx \mathcal{T}$ and we use $Q_{f}(\mathrm{e} / 2, t-(\mathcal{T}-\langle\delta \tau\rangle))$ as the average charge dependence. The Fourier transform then takes the form:

$$
S(\omega)=N \mathcal{T}\left\langle|F(\omega)|^{2}\right\rangle \mathcal{A}(\omega),
$$

where

$$
F(\omega)=\frac{1}{N} \sum_{n=0}^{N-1} e^{i \omega t_{n}}
$$


and

$$
\mathcal{A}(\omega)=\left|e^{i \omega\langle\delta \tau\rangle} \int_{0}^{\mathcal{T}} \frac{d t}{\mathcal{T}} Q_{f}(\mathrm{e} / 2, t-\mathcal{T}+\langle\delta \tau\rangle) e^{i \omega t}\right|^{2} .
$$

The quantity $\mathcal{A}$ can be readily evaluated:

$$
\begin{aligned}
& \mathcal{A}(\omega)=\left(\frac{\mathrm{e} \tau_{s}}{2 \mathcal{T}}\right)^{2} \times \\
& \times\left|\frac{\kappa+1}{\omega \tau_{s}}\left(e^{i \omega \mathcal{T}}-1\right)-\frac{\kappa e^{-\langle\delta \tau\rangle / \tau_{s}}}{\omega \tau_{s}+i}\left(e^{i \omega \mathcal{T}}-e^{\mathcal{T} / \tau_{s}}\right)\right|^{2} .
\end{aligned}
$$

In order to proceed we have to evaluate also the average of $F(\omega)$. It is convenient to express the time at which one event happens as a sum over the delays between previous events using Eq. (12):

$$
F(\omega)=\frac{e^{i \omega t_{0}}}{N}\left(1+\sum_{n=1}^{N-1} \exp \left\{i \omega \sum_{k=0}^{n-1} \mathfrak{F}\left(\delta \tau_{k}\right)\right\}\right) .
$$

Now the average of $|F(\omega)|^{2}$ can be performed using the distribution function $\mathcal{P}(\delta \tau)$ :

$$
\left\langle|F(\omega)|^{2}\right\rangle=\frac{1}{N}\left(1+2 \operatorname{Re}\left\{\frac{g(\omega)}{1-g(\omega)}\right\}\right)+\frac{\delta F}{N^{2}}
$$

where we introduce the quantities:

$$
g(\omega)=\left\langle e^{i \omega \mathfrak{F}(\delta \tau)}\right\rangle=\int_{0}^{\infty} d(\delta \tau) \mathcal{P}(\delta \tau) e^{i \omega \mathfrak{F}(\delta \tau)}
$$

and $\delta F=2 \operatorname{Re}\left\{g\left(g^{N}-1\right) /(1-g)^{2}\right\}$, whose contribution to $S(\omega)$ vanishes in the limit $N \rightarrow \infty$. In conclusion we find:

$$
S(\omega)=\left(1+2 \operatorname{Re}\left\{\frac{g(\omega)}{1-g(\omega)}\right\}\right) \mathcal{T} \mathcal{A}(\omega),
$$

which constitutes the central result of this section.

We are now in the position to study the spectrum of the charge fluctuations for the system at hand. As it can be seen from the form of (34) the function has a singularity for $g \rightarrow 1$. Since

$$
|g(\omega)|^{2}=\int_{0}^{\infty} d t \int_{0}^{t} d t^{\prime} \mathcal{P}(t) \mathcal{P}\left(t^{\prime}\right) 2 \cos (\omega t) \leq 1,
$$

a singularity is present for $\omega \rightarrow 0$ or when the fluctuations are negligible so that $g \approx e^{i \omega\langle\mathfrak{F}(\delta \tau)\rangle}$. This picture predicts a series of peaks for the frequencies $\Omega_{n}=2 \pi n / \mathcal{T}$ with $n$ integer. Small fluctuations introduce a finite width, regularizing the correlation function. The numerical integration in the expression of $g(\omega)$ is straightforward, but it is also possible to obtain an analytical expression. Deep in the SETOs regime $\mathcal{P}(\delta \tau)$ has a gaussian behavior and provides a short cutoff time, so that we can expand the exponential in (35) to second order in $\delta \tau$. This gives

$$
\begin{aligned}
& g(\omega)=e^{i \omega t_{\star}} \times \\
& \times\left(1+\frac{2 i \omega}{2+\kappa}\langle\delta \tau\rangle-\frac{\kappa^{2} i \omega+4 \omega^{2} \tau_{s}}{2(2+\kappa)^{2}} \frac{\left\langle\delta \tau^{2}\right\rangle}{\tau_{s}}+\ldots\right) .
\end{aligned}
$$

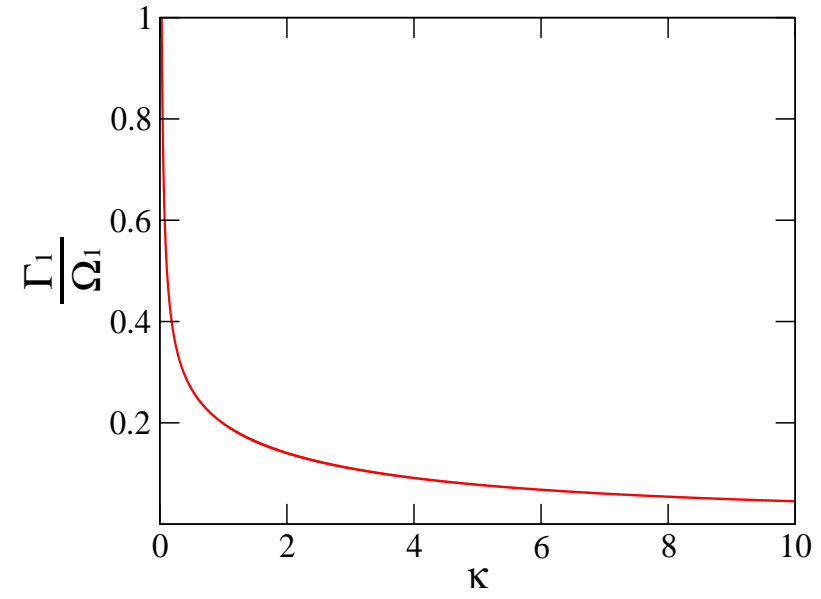

FIG. 8. The relative width at half height of the first noise peak as a function of $\kappa$, given by Eq. (43), for $\rho=10$.

The maximum of $\operatorname{Re}\{g /(1-g)\}$ takes place for $\operatorname{Arg}[g(\omega)]=0$ that at lowest order in $\langle\delta \tau\rangle$ gives for the position of the poles:

$$
\Omega_{n}=\frac{2 \pi n}{t_{\star}}\left(1-\frac{2\langle\delta \tau\rangle}{(2+\kappa) t_{\star}}\right),
$$

coinciding at linear order in $\langle\delta \tau\rangle / \tau_{s}$ with $2 \pi n / \mathcal{T}$. The phase of $g\left(g=|g| e^{i \phi}\right)$ thus vanishes at the minimum of $\operatorname{Re}\{g /(1-g)\}$, so that near this point one can write at lowest order $\phi \approx \mathcal{T}\left(\omega-\Omega_{n}\right)$. The relevant part of (34) then reads:

$$
\operatorname{Re}\left\{\frac{g}{1-g}\right\} \approx \frac{(1-|g|)}{(1-|g|)^{2}+\phi^{2}},
$$

and Eq. (29) takes the simple Lorentzian form

$$
S(\omega) \simeq \mathcal{A}\left(\Omega_{n}\right) \frac{\Gamma_{n} / 2}{\Gamma_{n}^{2} / 4+\left(\omega-\Omega_{n}\right)^{2}},
$$

with the full width at half maximum $\Gamma_{n}$ defined by:

$$
\Gamma_{n}=2 \frac{1-|g|}{\mathcal{T}}=\frac{4 \Omega_{n}^{2}}{\mathcal{T}(2+\kappa)^{2}}\left(\left\langle\delta \tau^{2}\right\rangle-\langle\delta \tau\rangle^{2}\right) .
$$

The presence in this formula of the mean squared variance of delay in the tunneling time, $\left\langle(\delta \tau-\langle\delta \tau\rangle)^{2}\right\rangle$, clearly indicates that the spread in the hopping events controls the width of the peak, as is physically expected. One also sees that the width of the poles increases with $n$. Performing the average with the gaussian distribution we find $\left\langle(\delta \tau-\langle\delta \tau\rangle)^{2}\right\rangle / \tau_{s}^{2}=4(1-\pi / 4) /(\kappa \rho)$ and for the relative width $\Gamma_{n} / \Omega_{n}$ we have the explicit expression:

$$
\frac{\Gamma_{n}}{\Omega_{n}}=\frac{32 \pi n(1-\pi / 4)}{\rho \kappa(2+\kappa)^{2} \ln ^{2}[(2+\kappa) / \kappa]} .
$$

Eq. (43) allows to study the width of the peak in the charge-fluctuation spectrum, and thus, the accuracy of 
the SETOs. It correctly gives that $\Gamma_{1} /(2 \pi / \mathcal{T}) \ll 1$ in the SETOs regime $(1 / \rho \ll \kappa \ll \rho)$. It also shows (see Fig. (8)) that the relative width $\Gamma_{1} / \Omega_{1}$ is a monotonic decreasing function of the bias current $(\kappa)$ for a given value of the resistance $(\rho)$.

From Eq. (41) it is clear that within this approximation the weight of the Lorentzian peak is controlled only by the form factor $\mathcal{A}\left(\Omega_{n}\right)$. Using its explicit expression one finds that for large $\rho$ it becomes independent of $\rho$ : $\mathcal{A}\left(\Omega_{1}\right) \simeq \mathrm{e}^{2} /\left(4 \pi^{2}+\ln ^{2}\left[\frac{2+\kappa}{\kappa}\right]\right)$. The full variation takes place in the $\kappa<1$ region, where the shape of $Q(t)$ evolves from the shark-fin to the sawtooth form. From the form of Eq. (43) one can also see that for small $\kappa$ the peak broadens and the SETOs are washed out when $\Gamma_{1} \sim \Omega_{1}$ (for $\kappa \sim 1 / \rho$ ). For large $\kappa$ the theory instead predicts that the relative width decreases monotonically [38]:

$$
\frac{\Gamma_{n}}{\Omega_{n}}=\frac{8 \pi n(1-\pi / 4)}{\rho \kappa} .
$$

In this limit the SETOs disappear by a decrease of the weight of the peak, but within our approximation this is not seen. Actually for sufficiently large current bias $(\kappa \sim \rho)$ there is a finite probability that a single tunnel event is no more sufficient to bring the charge back in the Coulomb blockade region. This is quantified by the value of $P_{n}\left(t_{n}\right)$, which, contrary to our hypothesis, can become smaller than 1 . One can expect the theory to roughly remain valid for the fraction of tunneling events that leaves $Q\left(t_{n}\right)<\mathrm{e} / 2$. This describes a peak that remains sharp, but that vanishes in weight as $P_{n}\left(t_{n}\right)$.

Another interesting and relevant limit is the lowfrequency behavior of $S(\omega)$. Expanding Eq. (35) in $\omega$ one can show that

$$
\begin{aligned}
N\left\langle|F(\omega)|^{2}\right\rangle & =\frac{F_{2}-F_{1}^{2}}{F_{1}^{2}}+ \\
& +\omega^{2} \frac{4 F_{1} F_{2} F_{3}-F_{1}^{2} F_{4}-3 F_{2}^{3}}{12 F_{1}^{4}}+\ldots
\end{aligned}
$$

where $F_{n}=\left\langle\mathfrak{F}^{n}\right\rangle$. If the fluctuations are negligible then $F_{n}=F_{1}^{n}$ and the noise at low frequency vanishes. In particular in our case this average takes a simple form if the explicit expression of $\mathfrak{F}$ is used:

$$
N\left\langle|F(\omega)|^{2}\right\rangle \simeq 4 \frac{\left\langle\delta \tau^{2}\right\rangle-\langle\delta \tau\rangle^{2}}{t_{\star}^{2}(2+\kappa)^{2}}\left(1+\frac{\omega^{2} t_{\star}^{2}}{12}+\ldots\right) .
$$

We thus find that in the SETOs regime the low-frequency noise is suppressed. Eq. (46) together with the expansion of Eq. (32) for $\omega \rightarrow 0$ allows us to evaluate the zero-frequency Fano factor: $\mathbb{F} \equiv S_{J}(0) / \mathrm{e}\left\langle I_{J}\right\rangle=$ $S(0) / \tau_{s}^{2} \mathrm{e}\left\langle I_{J}\right\rangle$, where $S_{J}(\omega)$ is the noise spectrum of the current through the junction and the relation $S(\omega)=$ $S_{J}(\omega) \tau_{s}^{2} /\left(1+\omega^{2} \tau_{s}^{2}\right)$ holds exactly. The reduction of the current fluctuations in the large- $\rho$ limit naturally leads to sub-Poissonian noise $(\mathbb{F}<1)$ vanishing for $\rho \rightarrow \infty$ :

$$
\mathbb{F} \simeq \frac{(4-\pi)\left(-2+(\kappa+1) t_{\star} / \tau_{s}\right)^{2}}{\rho \kappa(2+\kappa)^{2}\left(t_{\star} / \tau_{s}\right)^{2}} .
$$

Nevertheless this expression is only qualitatively correct, since the analytical theory has been designed to describe accurately the noise for frequencies around the peak of the SETOs. This will be shown in the next section, where we present numerical simulations.

\section{NUMERICAL SIMULATIONS}

In this section we show numerical results obtained by Monte Carlo simulations for the charge-fluctuation spectrum. The purpose of this section is to compare these results with the analytical calculations of the preceding sections, valid for $\rho \gg 1$ and $1 / \rho \ll \kappa \ll \rho$, and to explore the crossover region where the SETOs disappear.

The Monte Carlo simulations are performed by generating different realizations of the stochastic time evolution of the charge $Q(t)$ over a time much longer than the SETOs period. The time evolution of the charge is obtained by discretizing the time on a nonuniform grid, such that in the time interval $\Delta t$ the charge varies by a small quantity and $P=\Gamma(Q(t)) \Delta t<\mathcal{N} \ll 1$. The tunneling event is accepted or refused by generating a random number between 0 and 1 and by comparing it to $P$. The deterministic evolution of $Q(t)$ between two events is simply given by Eq. (3). The sequence of time intervals of deterministic evolution interspersed by tunneling times so constructed gives the full knowledge of $Q(t)$ and constitutes the stochastic run. The square modulus of the Fourier transform of the charge is then easily calculated analytically piecewise, interval by interval. To obtain the noise as from (27), just a further average over several runs is needed. Typically $\mathcal{N}=.01$, each run counts $10^{3}$ tunneling events, and an average over $10^{4}$ realizations is performed.

Let us now discuss the numerical results. We begin by comparing the form of the first peak in the noise spectrum. It is shown scaled by the analytically calculated width $\Gamma_{1}$ in Fig. (9) for different values of $\rho$ and given $\kappa$. The agreement is excellent. We then compare the full $\omega$ dependence of $S(\omega)$ obtained from Eq. (36) with the one calculated numerically. We show the comparison in Fig. (10) for the relevant case $\kappa=1$ (which corresponds to the widest extension in $\rho$ of the SETOs region) and for $\rho$ ranging from 200 to 10 . As expected, the agreement is very good deep in the SETOs regime, for $\rho \gg 1$, and at $\rho=10$ all the essential features of the peak at $\omega=\Omega_{1}$ are still fairly well represented by the theory. The analytical approximation correctly finds the main contribution to the noise, but it tends to underestimate it far from the peak maximum.

Let us now investigate how the SETOs disappear. From the experimental point of view a simple parameter that can be varied continuously is $\kappa \sim I_{b}$. We thus plot in Fig. (11) the evolution of $S(\omega)$ for given $\rho=10$ and 100 as a function of $\kappa$. These two plots show several interesting features. The first striking one is the reduction of the relative widths of the peaks by increasing $\kappa$. 


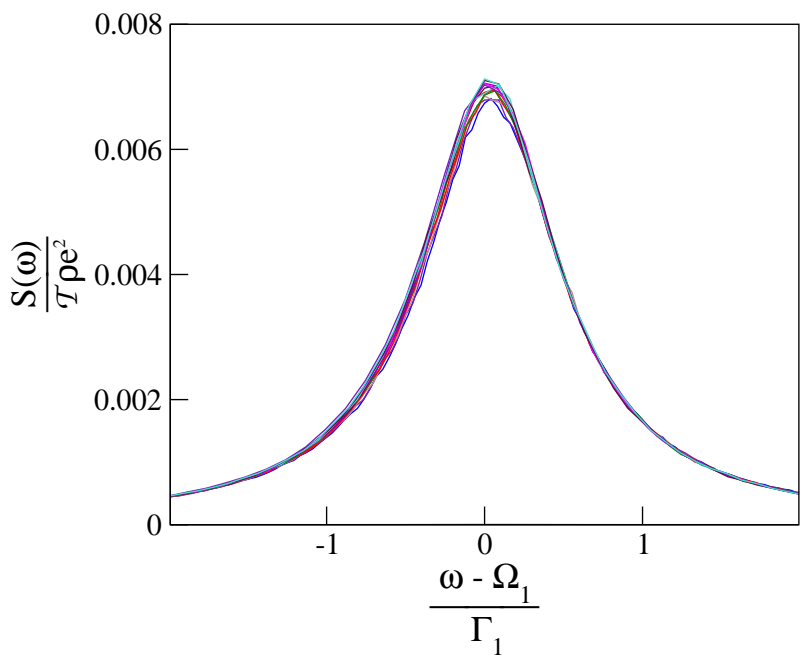

FIG. 9. (Color online) The noise peak at $\omega=\Omega_{1}$ obtained from Monte Carlo numerical simulations for values of $\rho$ ranging from 80 to 200 in steps of 10 . On the $x$-axis the frequency is shifted by $\Omega_{1}$ and scaled by the width $\Gamma_{1}$ as given by Eq. (42), on the $y$-axis the noise is scaled by $\mathcal{T} \rho$.
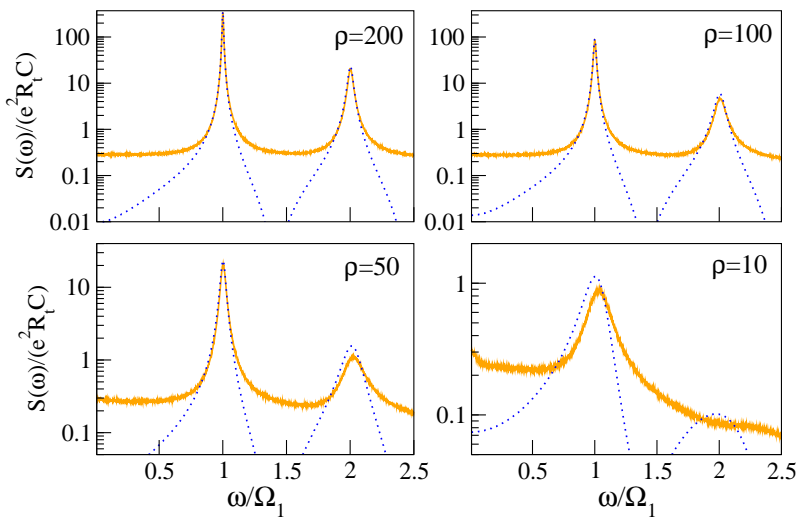

FIG. 10. (Color online) Comparison between Monte Carlo data for the charge-fluctuation spectrum and the analytical results obtained from Eq. (36) for different values of $\rho$, at fixed bias condition $\kappa=1$.

This is predicted by the analytical expression (43) and the numerical calculations assess its validity even outside the region of applicability of the analytical theory. Note that in Fig. (11) the frequency axis is rescaled with $\Omega_{1}$, thus the apparent weight of the peaks is reduced by the scaling, but it saturates in the region $\kappa \ll \rho$ as predicted by the analytical theory, and then starts to decrease in the crossover region. The second visible feature is the appearance of a wide Lorentzian zero frequency peak that remains the only structure for $\kappa \gg \rho$. This structure is due to the charge noise induced at the capacitance by the Poissonian current fluctuations generated by the tunnel junction. By solving the electromagnetic problem one finds: $S(\omega)=C^{2}|Z(\omega)|^{2} e\left\langle I_{J}\right\rangle$, where $e\left\langle I_{J}\right\rangle$ is the standard tunnel junction Poissonian white noise and $Z(\omega)=R_{\|} /\left(1+i \omega R_{\|} C\right)$, with $R_{\|}=R_{s} R_{t} /\left(R_{s}+R_{t}\right)$, the
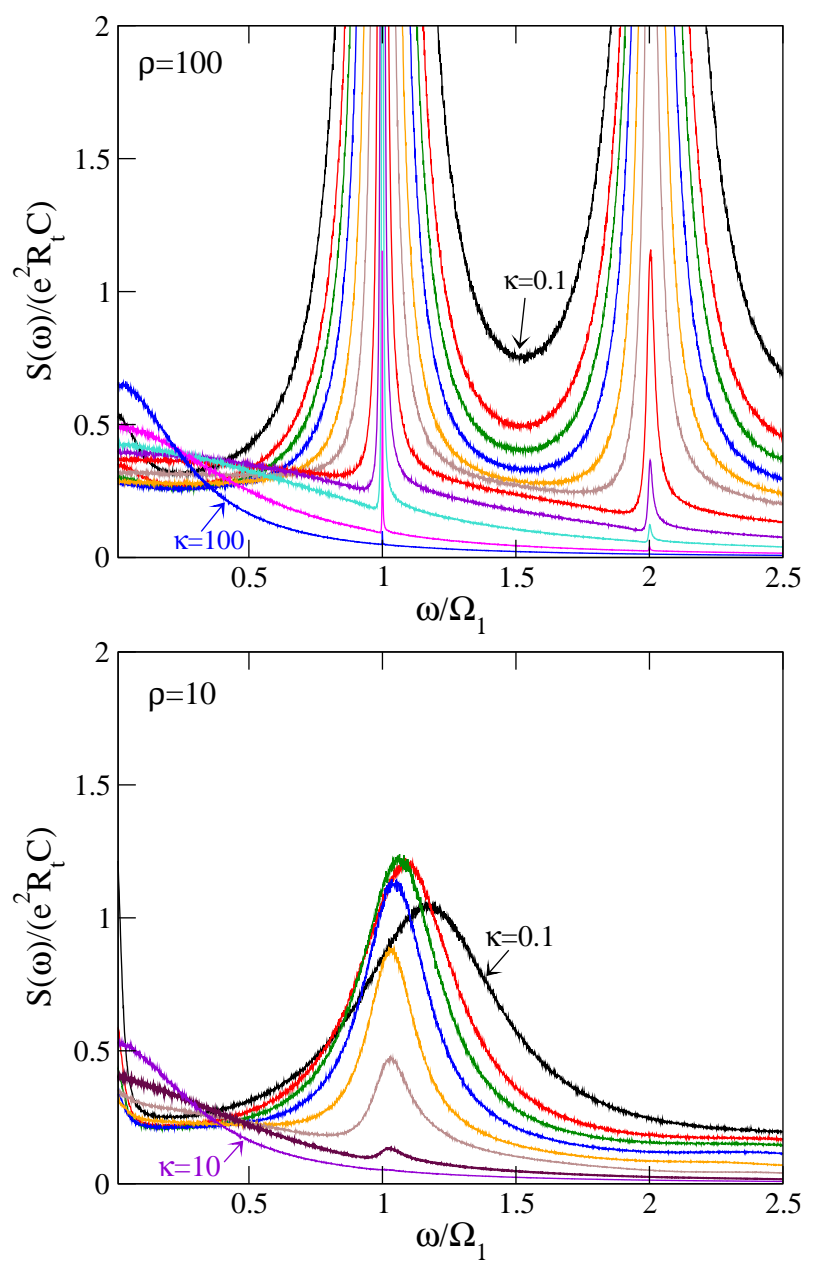

FIG. 11. (Color online) Monte Carlo spectra for different bias conditions $\kappa$ at fixed junction environment: $\rho=100$ $(\rho=10)$ in the upper (lower) graph. Increasing $\kappa$ means here moving along a horizontal line in Fig. (7) toward the highbias boundary of the SETOs region and it allows us to see how SETOs disappear.

impedance between the current source and the voltage at the capacitance. This gives:

$$
S(\omega)=\frac{\mathrm{e}\left\langle I_{J}\right\rangle C^{2} R_{s}^{2}}{1+2 \rho+\rho^{2}\left(1+\omega^{2} R_{t}^{2} C^{2}\right)},
$$

which fits our data very well (not shown).

Finally we show in Fig. (12) the evolution of $S(\omega)$ for $\kappa=1$ and for $\rho$ evolving from 1 to 100 . This figure gives an idea of the expected spectrum at the optimal value $\kappa=1$ as a function of $\rho$. It turns out that already at $\rho=3, S(\omega)$ presents a very broad maximum and $\rho=5$ is probably sufficient to observe a clear structure in $S(\omega)$.

\section{CONCLUSIONS}

In this paper we have studied theoretically electronic transport in a tunnel junction in the presence of a large- 


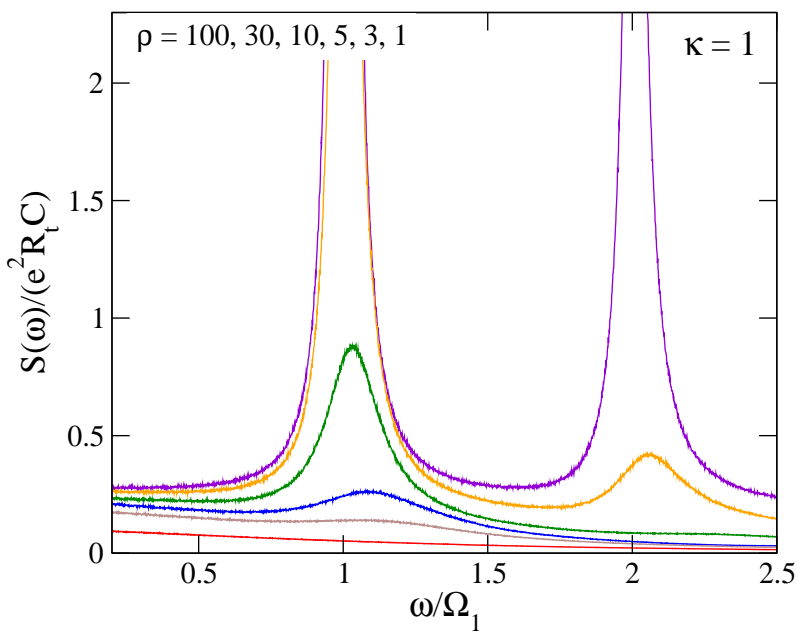

FIG. 12. (Color online) Monte Carlo spectra for fixed bias conditions $\kappa=1$ and different values of $\rho$ : The crossover from the SETOs regime to the $\rho \lesssim 1$ region is shown.

resistive environment. The phenomenon of SETOs has been predicted to appear in this system for essentially infinite value of the external resistance, so that the junction can be seen as current biased. We investigated under which conditions the SETOs appear for a realistic finite value of the environment resistance. We found analytical expressions for the current [Eq. (20)] and for the chargefluctuation spectrum [Eq. (29) with Eq. (42)]. Our analytical results describe very well the form of the peak in the charge noise, which can be regarded as the hallmark of the SETOs, since it quantifies the accuracy of the periodicity in the charge time dependence. We find that a ratio of $R_{s} / R_{t}$ as low as 5 can be sufficient to observe a clear structure in $S(\omega)$ if the bias current is chosen such that $I_{b} \approx 2 I_{\mathrm{th}}=\mathrm{e} / R_{s} C$ (this can be converted on a condition on the voltage bias $\left.V_{b} \approx \mathrm{e} / C\right)$. This ratio can be obtained experimentally and thus in principle SETOs can be observed through the measurement of the current or charge noise.

The conclusion on the possibility of observing the effect is thus optimistic. One should however keep in mind that, following the literature on this problem, the theory presented holds at low temperature $\left(k_{B} T \ll \mathrm{e}^{2} / 2 C\right)$ and neglects quantum fluctuations of the electromagnetic modes of the environment. Thermal and quantum fluctuations are expected to have a non-negligible influence on the transport mechanism at play in the system. Their evaluation requires a different technical approach and is beyond the scope of the present paper.

\section{ACKNOWLEDGEMENTS}

We are indebted for useful correspondence with Y. Pashkin. We also gratefully acknowledge fruitful discussions with D. Esteve, F. Portier, and L.S. Kuzmin. Comments on the manuscripts are acknowledged from
R. Avriller, V. Puller, and M. Houzet. We finally acknowledge partial financial support through the French ANR grant QNM No. 040401.

\section{Appendix A: CALCULATION OF THE $I-V$ CHARACTERISTICS IN THE SETOS REGIME WITH THE MASTER EQUATION APPROACH}

In this Appendix we find for the $I-V$ characteristics expressions which take into account the spread in the distribution probability of the charge. A full statistical description of the behavior of the system can be given in terms of the probability $\sigma_{n}(Q, t)$ that at time $t$ the charge in the capacitance is $Q$ and $n$ charges have crossed the junction. Conservation of the probability and the master equation describing electron tunneling lead to the following set of coupled partial differential equations:

$$
\begin{aligned}
& \frac{\partial \sigma_{n}(Q, t)}{\partial t}=\frac{\partial}{\partial Q}\left[\frac{\left(Q-Q_{s}\right)}{\tau_{s}} \sigma_{n}(Q, t)\right] \\
& -\Gamma(Q / C) \sigma_{n}(Q, t)+\Gamma((Q+\mathrm{e}) / C) \sigma_{n-1}(Q+\mathrm{e}, t) .
\end{aligned}
$$

Eq. A1 is a generalization of the equations given in Ref. [7] to include the information on the number of electrons which have tunneled. The general solution of this equation is difficult in the presence of a finite resistance. But in the SETOs regime we can find an explicit solution by exploiting the fact that at every cycle the charge passes through the blocked range $(-\mathrm{e} / 2<Q<\mathrm{e} / 2)$. Let us assume that at $t=0$ the distribution function is:

$$
\sigma_{n}(Q, 0)=\delta(Q-\mathrm{e} / 2) \delta_{n, n_{0}} .
$$

The differential equation (A1) for $\sigma_{n_{0}}(Q, t)$ can be easily solved, since it decouples from the others $\left(\sigma_{n_{0}-1}=0\right)$ :

$$
\sigma_{n_{0}}(Q, t)=P(t) \delta\left(Q-Q_{f}\left(\frac{\mathrm{e}}{2}, t\right)\right)
$$

where $P(t)=P_{n}(t)$ as given by Eq. (16) with $t_{n}=0$ and $Q_{f}$ is defined in Eq. (3). Once we know the solution for $\sigma_{n_{0}}$ we can substitute it into Eq. A1 and find the solution for $\sigma_{n_{0}+1}$. This can be done by using the ansatz:

$$
\sigma_{n_{0}+1}(Q, t)=\int d Q^{\prime} \delta\left(Q-Q_{f}\left(Q^{\prime}, t\right)\right) f\left(Q^{\prime}, t\right),
$$

that gives

$$
\sigma_{n_{0}+1}(Q, t)=f(z(Q), t) e^{\frac{t}{\tau_{s}}}
$$

with $z(Q)=\left(Q-Q_{s}\right) e^{\frac{t}{\tau_{s}}}+Q_{s}$, and $f(z(Q), t)=0$ for $z(Q)<\mathrm{e} / 2$ and $t>\tau_{s} \ln \left(\frac{1}{2}-\frac{z(Q)}{\mathrm{e}}\right)$. The resulting differential equation for $f$ reads:

$$
\begin{aligned}
& \frac{\partial f(z(Q), t)}{\partial t}=\left(Q_{s}+\frac{\mathrm{e}}{2}+\left(z(Q)-Q_{s}\right) e^{-\frac{t}{\tau_{s}}}\right) \times \\
& \times e^{-\frac{t}{\tau_{s}}} \frac{P(t)}{\mathrm{e} R_{t} C} \delta\left(\mathrm{e}+\left(z(Q)-\frac{\mathrm{e}}{2}\right) e^{-\frac{t}{\tau_{s}}}\right) .
\end{aligned}
$$


The equation can be integrated and gives for $\sigma_{n_{0}+1}(Q, t)$

$$
\begin{aligned}
\sigma_{n_{0}+1}(Q, t) & =e^{\frac{t}{\tau_{s}}} P\left(\tau_{s} \ln \left(\frac{1}{2}-\frac{z(Q)}{\mathrm{e}}\right)\right) \times \\
& \times \frac{\tau_{s}}{\mathrm{e} R_{t} C} \frac{\left(\frac{\mathrm{e}}{2}-Q_{s}\right)\left(z(Q)+\frac{\mathrm{e}}{2}\right)}{\left(z(Q)-\frac{\mathrm{e}}{2}\right)^{2}}
\end{aligned}
$$

that is non vanishing for $Q_{1}(t)<Q<Q_{2}(t)$ where $Q_{1}(t)=Q_{s}-\mathrm{e}-\left(Q_{s}-\mathrm{e} / 2\right) e^{-t / \tau_{s}}$ and $Q_{2}(t)=Q_{s}-$ $\left(Q_{s}+\mathrm{e} / 2\right) e^{-t / \tau_{s}}$. It is interesting to note that the distribution has now a finite spread in $Q$ induced by the combined action of the stochastic fluctuations and of the finite value of the resistance. This is in contrast with the simpler approximation used in the text to evaluate the average in Eq. (20), where we assumed that the spread was negligible, and that a delta function could be used to describe the distribution $\sigma_{n_{0}+1}$.

We can now calculate the average charge on the junction during a single oscillation:

$$
\langle Q\rangle=\int_{0}^{\mathcal{T}} \frac{d t}{\mathcal{T}}\left(Q_{f}\left(\frac{\mathrm{e}}{2}, t\right) P(t)+\int_{Q_{1}(t)}^{Q_{2}(t)} d Q Q \sigma_{n_{0}+1}(Q, t)\right)
$$

The numerical integration of this expression leads to the result shown in Fig. (6).
[1] G. L. Ingold and Yu. V. Nazarov, in Single Charge Tunneling, Vol. 294 of NATO ASI Series B: Physics, edited by H. Grabert and M. Devoret (Plenum, New York, 1992).

[2] H. Grabert, G. L. Ingold, M. H. Devoret, D. Esteve, H. Pothier and C. Urbina, Z. Phys. B: Condensed Matter 84, 143 (1991).

[3] D. Popović, C. J. B. Ford, J. M. Hong and A. B. Fowler, Phys. Rev. B 48, 12349 (1993).

[4] F. Pierre, H. Pothier, P. Joyez, N. O. Birge, D. Esteve, M. H. Devoret, Phys. Rev. Lett 86, 1590 (2001).

[5] T. T. Hongisto, A. B. Zorin, arXiv:1109.3634v1.

[6] E. Ben-Jacob, Y. Gefen, Phys. Lett. A 108, 289 (1985).

[7] D. V. Averin and K. K. Likharev, J. Low Temp. Phys. 62, 345 (1986).

[8] D. V. Averin, K. K. Likharev, IEEE Trans. Magn. 23, 1138 (1987).

[9] K. K. Likharev, IBM J. Res. Dev. 32, 144 (1988).

[10] J. M. Martinis, R. L. Kautz, Phys. Rev. Lett. 63, 1507 (1989).

[11] L. S. Kuzmin, Yu. V. Nazarov, D. B. Haviland, P. Delsing, T. Claeson, Phys. Rev. Lett. 67, 1161 (1991).

[12] A. N. Cleland, J. M. Schmidt, J. Clarke, Phys. Rev. Lett. 64, 1565 (1990).

[13] A. N. Cleland, J. M. Schmidt, J. Clarke, Phys. Rev. B 45, 2950 (1992).

[14] L. S. Kuzmin, Yu. A. Pashkin, Physica B 194, 1713 (1994).

[15] P. Joyez, D. Esteve, M. H. Devoret, Phys. Rev. Lett. 80, 1956 (1998).

[16] W. Zheng, J. R. Friedman, D. A. Averin, S. Han, J. E. Lukens, Solid State Commun. 108, 839 (1998).

[17] P. Delsing, K. K. Likharev, L. S. Kuzmin, T. Claeson, Phys. Rev. Lett. 63, 1861 (1989).

[18] P. Delsing, T. Claeson, K. K. Likharev, L. S. Kuzmin, Phys. Rev. B 42, 7439 (1990).

[19] K. K. Likharev, N. S. Bakhvalov, G. S. Kazacha, S. I. Serdyukova, IEEE Trans. Magn. 25, 1436 (1989).

[20] J. Bylander, T. Duty, and P. Delsing, Nature 434, 361 (2005).
[21] K. K. Likharev, A. B. Zorin, J. Low Temp. Phys. 59, 347 (1985).

[22] L. S. Kuzmin, D. B. Haviland, Phys. Rev. Lett. 67, 2890 (1991).

[23] L. S. Kuzmin, IEEE Trans. Magn. 3, 1983 (1993).

[24] S. Corlevi, W. Guichard, F. W. J. Hekking, and D. B. Haviland, Phys. Rev. Lett. 97, 096802 (2006).

[25] I. S. Beloborodov, F. W. J. Hekking, F. Pistolesi in New Directions in Mesoscopic Physics (Towards Nanosciences), p. 339, R. Fazio, V. F. Gantmakher and Y. Imry ed., (Kluwer Academic Publishers, New York, 2003).

[26] M. Watanabe, D. B. Haviland, R. L. Kautz, Supercond. Sci. Technol. 14, 870 (2001).

[27] M. Watanabe and D. B. Haviland, Phys. Rev. Lett. 86, 5120 (2001).

[28] S. Corlevi, W. Guichard, F. W. J. Hekking, and D. B. Haviland, Phys. Rev. B 74, 224505 (2006).

[29] F. Maibaum, S. V. Lotkhov, and A. B. Zorin, Phys. Rev. B 84, 174514 (2011).

[30] R. Aguado, L. P. Kouwenhoven, Phys. Rev. Lett. 84, 1986 (2000).

[31] E. Zakka-Bajjani, J. Ségala, F. Portier, P. Roche, D. C. Glattli, A. Cavanna, Y. Jin, Phys. Rev. Lett. 99, 236803 (2007).

[32] J. Gabelli, B. Reulet, Phys. Rev. Lett. 100, 026601 (2008).

[33] J. Basset, H. Bouchiat, R. Deblock, Phys. Rev. Lett. 105, 166801 (2010).

[34] M. Ueda, Phys. Rev. B 42, 3087 (1990).

[35] M. Ueda, Y. Yamamoto, Phys. Rev. B 41, 3082 (1990).

[36] M. Ueda, N. Hatakenaka, Phys. Rev. B 43, 4975 (1991).

[37] G. Schön and A. D. Zaikin, Phys. Rep. 198, 237 (1990).

[38] This expression does not agree with the expression (57) of Ref. 7], specifically we find a different functional dependence on $R_{s}: \Gamma \sim 1 / R_{s}^{2}$ instead of $1 / R_{s}$. 